\title{
Improving the interface between family planning services and genitourinary medicine in Portsmouth, UK: a 'virtual corridor'
}

\author{
Alison Blume, Sally Kidsley, Jean Tobin, Graham Davies
}

\begin{abstract}
Background and methods In locations where the genitourinary medicine (GUM) department and the family planning services (FPS) are on separate sites, studies have shown a low rate of attendance of clients referred between the sites. We developed a coupon system to allow clients referred from one site to be seen without a wait in the open-access clinic at the other site. Data from the first 5 months were collected.
\end{abstract}

Results A total of 59 clients were referred from the FPS to GUM during the time period studied. Of these, 54 (91.5\%) attended the GUM clinic. The majority (67\%) were referred with symptoms suggestive of a sexually transmitted infection (STI), while other reasons for referral included contacts of STI, high-risk behaviour, and following a sexual assault. Some $40 \%$ of clients were seen within 30 minutes of their referral. Of clients referred from GUM to FPS, $12 / 18$ (67\%) attended the clinic. This is significantly lower than the attendance of clients referred to $\operatorname{GUM}(p=0.016)$. For GUM to FPS referrals, $63 \%$ were seen within 30 minutes of their referral. Seven clients were referred for consideration of intrauterine device insertion for emergency contraception but only four attended. Similarly, of the four clients referred who were not using any contraception only one attended.

Discussion and conclusions We believe the coupon system helped increase attendance rates of clients referred between the two clinics, especially by eliminating the wait in the second clinic. The lower rate of attendance in those referred for contraception is of concern.

Keywords client referral, family planning service provision, genitourinary medicine, sexually transmitted infection

J Fam Plann Reprod Health Care 2007; 33(2): 113-115 (Accepted 30 June 2006)

\section{Background}

In a number of settings in the UK, an holistic approach to treatment of sexual health problems has been developed with clinics jointly run by genitourinary medicine (GUM) and family planning services (FPS). 1,2 These clinics provide the clients with an opportunity to discuss both contraceptive and sexual health needs in one visit. ${ }^{3}$ By providing this facility the detection, treatment and contact tracing of sexually transmitted infections (STIs) are improved. The likelihood of re-attendance and successful treatment has also been shown to increase. ${ }^{2}$

In locations where the GUM department and FPS are on separate sites there is a lower rate of attendance of clients with positive STI results referred from FPS to GUM. ${ }^{4}$ A wide variation has been shown in the length of time between referral from a family planning clinic to attendance at the GUM department, which may span weeks. ${ }^{1}$ Studies have shown that one in five women attending a family planning clinic have symptoms or concerns regarding an STI, which may indicate that these women feel more comfortable attending a family planning setting to deal with their concerns. ${ }^{4}$

Where the two departments are located on separate sites there may be methods of reducing the waiting time of clients who are referred between the clinics, thus increasing the likelihood of attendance and therefore treatment. In Portsmouth, UK, the GUM department is

Department of Genitourinary Medicine, St Mary's Hospital, Portsmouth, UK

Alison Blume, MRCP, DipGUM, Specialist Registrar

Jean Tobin, FRCOG, FRCP, Consultant

Ella Gordon Unit, St Mary's Hospital, Portsmouth, UK

Sally Kidsley, DFFP, MRCOG, Registrar

Graham Davies, MD, FRCOG, Consultant

Correspondence to: Dr Alison Blume, Department of Genitourinary Medicine, St Mary's Hospital, Milton Road, Portsmouth PO3 6AD, UK. E-mail: alison.blume@porthosp.nhs.uk

\section{Key message points}

- A coupon system has facilitated referral of clients between family planning services and genitourinary medicine clinics by eliminating the wait for clients in the second clinic.

- Attendance was significantly lower in clients referred for contraception needs than those referred with a possible sexually transmitted infection.

located 100 metres from the main family planning clinic, the Ella Gordon Unit (EGU), with overlap of opening hours. Some clients attending the EGU have sexual health problems more appropriately managed by the GUM department, and clients requesting contraceptive advice may attend GUM. In the past such clients were given a list of opening times of the alternative department but few were thought to have attended. In order to address this problem a fast-track system was set up. This 'virtual corridor' between the clinics allowed clients attending one of the units but considered to need urgent referral to the other unit to be seen with minimal delay. The aim of the 'virtual corridor' was to increase attendance rates at the more appropriate service for clients presenting with contraception problems or requiring STI screening and treatment.

\section{Methods}

A referral slip was designed with space for client details, date, time and brief reasons for referral. The slip was perforated, allowing one half to be given to the client and the other half to remain in the referring department. The client was informed verbally of the opening times and location of the other clinic and told that they would not have to wait in line to be seen.

On arrival at the clinic the slip was given to the receptionist, and the client was placed at the front of the queue for the walk-in service. As the client was seen the clinician wrote the date and time on to the slip.

Prior to the commencement of the audit both departments had agreed on suitable referral criteria. 
From EGU to GUM these were a positive chlamydia test in a client requesting a full STI screen, symptoms suggestive of a STI whose management was beyond the remit of the EGU, and high-risk clients who were deemed unlikely to return for a booked appointment. From GUM to EGU the referral criteria were requirement for an intrauterine device (IUD) as emergency contraception, and high-risk clients for immediate contraceptive advice.

The number of slips presented at each clinic was compared with the number of referrals made. Details of any referred client who had not handed in their slip were crosschecked as to whether they had been seen in the clinic.

The notes of all clients referred and seen were reviewed to find the reason for and outcome of the referral.

\section{Statistics}

Fisher's exact test was used to assess whether there was an association between the rate of attendance in clients referred to GUM and those referred to EGU. SPSS 13.0 for Windows (SPSS Inc., Chicago, IL, USA) was used for statistical analysis, while confidence intervals and Fisher's exact test were derived from the web calculator found at http://statpages.org.

\section{Results}

The study ran for 5 months from 3 May 2005 to 30 September 2005. Three variables were analysed as follows: 1. Time between end of consultation in one unit and start of consultation in the other unit.

2. Number of clients failing to attend following referral.

3. Appropriateness of referrals.

\section{Referrals from EGU to GUM}

During the 5-month study period 112 clients were diagnosed and treated for a STI wholly within the EGU. A further 59 clients were referred from EGU to GUM via the "virtual corridor'. Of these, 54 (91.5\%, 95\% CI 81.3\%-97.2\%) had documented evidence of attending the department.

The average age of those clients seen was 24.1 (range, $14-59)$ years and two were aged under 16 years. Only 5/54 $(9.3 \%)$ of the clients seen were male.

The majority of clients were referred with symptoms suggestive of a STI $(36 / 54,66.7 \%)$. Other reasons for referral are given in Table 1.

Of the clients for whom both the time referred and the time seen were documented $(n=40), 16(40 \%)$ were seen within 30 minutes of referral. The median time from referral to being seen was 40 minutes. However, much of this time was due to a delay in presenting to the GUM department. The median wait within the GUM department was 14 minutes (interquartile range, $7.5-25.5$ minutes), with $36 / 41(87.8 \%)$ of clients waiting less than 30 minutes

Table 1 Reasons for referral to the genitourinary medicine department

\begin{tabular}{lrc}
\hline Reason for referral & $\boldsymbol{n}$ & $\%$ \\
\hline Chlamydia positive & 1 & 1.9 \\
Contact of STI & 4 & 7.4 \\
Symptoms of STI & 36 & 66.7 \\
High risk of STI & 5 & 9.3 \\
HIV test & 2 & 3.7 \\
Other & 3 & 5.6 \\
None given & 3 & 5.6 \\
Total & 54 & $(100.0)$ \\
\hline
\end{tabular}

STI, sexually transmitted infection.
Table 2 Reasons for referral to the family planning clinic (Ella Gordon Unit)

\begin{tabular}{lcc}
\hline Reason for referral & $\boldsymbol{n}$ & $\%$ \\
\hline Emergency contraception & 7 & 38.9 \\
No contraception & 4 & 22.2 \\
Running out of OCP & 1 & 5.6 \\
IUD problem & 3 & 16.7 \\
Other & 1 & 5.6 \\
None given & 2 & 11.1 \\
Total & 18 & $(100.0)$ \\
\hline
\end{tabular}

IUD, intrauterine device; OCP, oral contraceptive pill.

A total of $11(20.4 \%)$ clients had contact tracing initiated within the GUM department. Twenty (40.8\%) clients had diagnoses not caused by a STI. One client received epidemiological treatment as a contact of chlamydia although tests were subsequently negative, and one client was commenced on post-exposure prophylaxis for HIV following a sexual assault. In total 37/54 (68.5\%) clients seen received some form of treatment from GUM. Seventeen $(31.5 \%)$ clients underwent STI testing only and had negative results.

\section{Referrals from GUM to EGU}

Eighteen clients were referred to the EGU from GUM via the 'virtual corridor' during the 5-month study period. Of these, $12(66.7 \%, 95 \%$ CI $41.0 \%-86.7 \%)$ were seen in the EGU and six did not attend. This is significantly lower than the attendance of clients referred to GUM $(p=0.016)$. The median age of clients referred was 21 (range, 14-34) years and all were female.

Of the 11 clients with the time referred and time seen documented, seven $(63.6 \%)$ were seen within 30 minutes of referral. The median time delay was 20 (interquartile range, 10-40) minutes. We were unable to document how long the clients actually waited within the EGU.

The reasons for referral are given in Table 2. Of the seven clients referred for emergency contraception (all outside the 72-hour period for oral postcoital contraception) only four attended the EGU. Likewise, of the four sexually active clients who were not using any form of contraception only one attended the clinic for advice.

\section{Discussion and conclusions}

The 'virtual corridor' was successful in ensuring clients presenting to EGU with proven or possible STIs were seen in GUM. In the present study, $91.5 \%$ of those referred attended the GUM department. This is considerably higher than has been shown in a previous study. 5

Many of the clients referred were symptomatic $(66.7 \%)$, which may help to explain the high attendance rate. Also the GUM department in Portsmouth is open for walk-in clients every day from 8.30 am until 2 hours prior to evening closing, meaning that the clinic was open at the time of referral for the majority of clients. The high rate of attendance may also be partly due to recent publicity regarding STIs, particularly chlamydia, with Portsmouth being one of the pilot regions for the National Chlamydia Screening Programme.

The 'virtual corridor' was less successful in the other direction, with significantly fewer of those referred from GUM actually attending the EGU $(12 / 18,66.7 \%$ as compared with $54 / 59,91.5 \%, p=0.016)$. Of particular concern was the fact that only four out of seven clients who were eligible for an IUD insertion as emergency contraception after an episode of unprotected sexual intercourse actually attended the clinic. This may be 
Family planning and GUM interface/Book reviews

partially explained by the different opening times of the EGU in Portsmouth, which is open for walk-in clients for 2 hours each morning and 2 hours most evenings. Thus some clients seen in GUM would have to wait several hours before the EGU would open.

Of four clients using no form of contraception and referred to the EGU only one attended the clinic. This highlights an area of concern as these clients had already taken the step of accessing a sexual health service but had not had their contraceptive needs addressed. With the Government initiative aiming to halve teenage conceptions by 2010 , it is important that women presenting to any sexual health service can have their contraceptive needs met.

In areas where the FPS and GUM are on separate sites, it is important to explore novel methods to increase the attendance rate of those referred between the clinics. A coupon system that eliminates the wait in the second clinic has facilitated the referral system in Portsmouth.
Statements on funding and competing interests

Funding None identified.

Competing interests None identified.

References

1 Melville CRS, Bigrigg A, Nandwani R. Client perspectives on sexual health service provision. Int J STD AIDS 2004; 15: 380-383.

2 Wilkinson C, Hampton N, Bradbeer C. The integration of family planning and genitourinary medicine services. Br J Fam Plann 2000; 26: 187-189.

3 Jones $M$. Integrating family planning with genitourinary medicine: developing an holistic sexual health clinic in Eastbourne. Br J Fam Plann 2000; 26: 221-224.

4 Siddiqui F, Kirkman RJ, Chandiok S. Re-audit of referral compliance of chlamydia positive women from a family planning clinic. J Fam Plann Reprod Health Care 2004; 30: 86-87.

5 Tobin JM, Bateman J, Banks B, Jeffs J. Clinical audit of the process of referral to genitourinary medicine of patients found to be chlamydia positive in a family planning service. Br J Fam Plann 1999; 24: 160-163.

BOOK REVIEWS

Fast Facts: Menopause (2nd edn). D Barlow, B Wren. Oxford, UK: Health Press Ltd, 2005. ISBN: 1-903734-38-X. Price: £15.00. Pages: 108 (paperback)

The 48-year-old pallid woman in front of you anxiously asks to have her hormones checked. A haemoglobin and ferritin are more immediately relevant. The menopause as a physiological stage often coincides with the development of other chronic diseases and this should be explored. By the time both patient and doctor are ready for specific information they should have got to know each other. It needs to be considered why the woman has presented, and what her expectations are.

Those in search of fast facts about the menopause have a lot of choice. Amazon has many titles to choose from. The Internet has many suitable sites; some are referenced at the back of this book. Several other books aimed at UK clinicians have preceded this one.

In just 103 pages this book covers, chapter by chapter, the common symptoms expressed by menopausal women. It also describes the types of hormone therapy (including tibolone), their side effects and risks as well as alternative treatments.

Refreshingly, the book does not take a partisan approach to hormone replacement therapy (HRT) but offers a rational résumé of the options available.

Written in a good style, the text flows sufficiently well to read at one sitting. For subjects like the Women's Health Initiative Study, the summary is easy to read and sensibly explained. Time is taken to describe symptoms carefully and systematically. Key points are clearly presented, and the book is attractively laid out. The illustrations break up the text well but some diagrams seem a little superfluous, as often happens in this style of publishing where a series of topics are presented in a unified pattern. Many doctors would do well to study the British National Formulary as first choice before prescribing HRT, but it is useful to have another short text available for reference. Sometimes referring to a book can be reassuring for the patient at the consultation, by providing an objective view. This book is a reasonable choice for GPs who do not wish to specialise in this area.

Frequently asked questions are presented in a useful table, though wisely the authors avoid the question of duration of use. Long-term users often have strong feelings when cessation is suggested! The book offers a discussion in general terms, together with a sensible synopsis of the evidence base.

Reviewed by Helen Grace Gibson, MRCGP, DFFP General Practitioner, Kingston-upon-Hull, UK

Understanding Menopause. K Ballard. Chichester, UK: John Wiley \& Sons Ltd, 2003. ISBN: 0-470-84471-X. Price: £8.99. Pages: 132 (paperback)

Karen Ballard is an academic medical sociologist with a special interest in women's health issues. This book is one of a series, mainly directed at patients, to increase the understanding of various health problems. It achieves its aim admirably. It is clearly written and set out in ten self-contained chapters. The chapters stand alone, and could be read in any order as there is cross-referencing and reiteration. As stated in the introduction, the book does not need to be read through but can be kept for reference. Although aimed at patients, I think it will be useful for medical students or health professionals new to the subject. Written in 2003 there are already a few things that are out of date, but this is inevitable with books. Throughout the text, areas of uncertainty and ongoing research are mentioned.

The first chapter encompasses biological changes during the menstrual cycle and menopause. This is mostly excellent. Whilst the text is accurate and clear, I cannot say the same about the line sketches. The drawings throughout are in the same style and add a light-hearted flavour to the book. Conversely, this style is not useful when describing the anatomy of the female genital tract. The second chapter gives a balanced account of symptoms of the menopause. The author draws on personal accounts from 'The Women's Health Study'. This was a recent postal survey of 650 women, with in-depth interviews in 32 women. I found it of interest that half the women surveyed reported a drop in libido after the menopause.

Further chapters deal competently with menorrhagia and fibroids. There is a good description of the types and means of hysterectomy, together with more cameo accounts from women about their experiences. These accounts help keep the book alive. Another chapter deals with the 'Change of Life', which is not seen to be the same thing as the menopause. We hear the voices of the women discuss the upheavals of mid-life, often coinciding with the menopause. Ballard then describes in detail health changes associated with the climacteric and gives good general preventative medicine advice. Not everyone would agree that hormone replacement therapy (HRT) protects against Alzheimer's disease, however.

Suddenly, in the middle of the book, the author describes how attitudes and theories around the menopause have changed throughout history. I am not quite sure why this entertaining chapter is here rather than at the beginning. Evidence of benefit for non-hormonal treatments is discussed in a refreshing manner. Ballard exaggerates the evidence for benefit from phytooestrogens in bone protection. Unlike many authors, Ballard discusses the side effects and drug interactions of alternative therapies. Chapters on HRT and contraception at the menopause are well-balanced and informative.

This is an easy book to read. It is balanced and would be helpful for women deciding whether to take HRT. I would have no reservations in recommending it to a patient and hope that it is updated soon. I will keep it on the shelf in my surgery and lend it out only to those patients or students whom I am sure will return it!

Reviewed by Margaret Denman, DFFP, IPM General Practitioner, Oxford, UK

\section{VOLUNTEERS NEEDED TO REVIEW BOOKS FOR THE JOURNAL}

The Journal regularly receives books for review and for this it relies on the services of a small team of expert reviewers. Whilst no payment is offered in respect of this role, reviewers do get to keep the books they review thus offering an opportunity to build up reviewer's own or their departmental book collection.

For further information please contact the Journal's Book Review Editor, Dr Kate Weaver via e-mail (kate.weaver@lpct.scot.nhs.uk). Please provide your contact details (mail and e-mail addresses), together with a note of any special interests and/or expertise. 